\title{
Biological therapies targeting arrhythmias: are cells and genes the answer?
}

\begin{abstract}
Introduction: The clinical presentation of arrhythmias ranges from mild symptoms such as dizziness, to life-threatening circulatory collapse. Current management includes medical therapy and procedures such as ablation or device implantation, however these strategies still pose a risk of side effects, while some patients remain symptomatic. Areas covered: Advancement in our understanding of how arrhythmias develop on the cellular level has made more targeted approaches possible. In addition, contemporary studies have found that several genes are involved in the pathogenesis of arrhythmias. Interestingly, gene and cell therapies allow treatments to be locally applied, bypassing systemic side effects in most occasions. Expert opinion: Pre-clinical studies have shown promising results in animal models of arrhythmias. However, more work is needed before this becomes a clinically viable option.
\end{abstract}

Keywords: genes, cells, arrhythmias, conduction

\section{Introduction}

The principle mechanisms of arrhythmias can be divided into disorders of impulse formation (triggered activity or automaticity) or impulse conduction (reentry)[1, 2]. Ion handling by the cardiomyocyte is a delicate process, the disruption of which can lead to disordered cardiac excitation and arrhythmias [3].

Genetic mutations can result in altered ion movement, even within a structurally normal heart. Changes to sodium, potassium and calcium handling distorts the cardiac action potential, resulting in altered depolarization or repolarization. Mutations, primarily within ion channel genes, underlie conditions such as Long QT Syndrome (LQTS) and Brugada [4], and less commonly bradyarrhythmias [5]. 
Most arrhythmias occur in the presence of structural heart disease where the cell structure has been altered, resulting in profound ion dysregulation [6]. Ischaemia converts conducting tissue into an electrically inactive scar, forcing the wave front into a region of slowconducting tissue, creating an environment suitable for reentry[2, 7]. Fibrosis occurs in pacemaker cells as part of the aging process, resulting in bradycardia [8].

Recent studies have used gene and cell therapy to alter impulse formation and impulse conduction properties. Genes of interest can be transferred using a viral or non-viral vector and delivered to the target area using direct injection or catheter-based techniques[9]. Gene transfer has been used to treat ventricular arrhythmias, bradycardia and atrial fibrillation within animal models[10]. Cell transplantation, generally embryonic or mesenchymal stem cells, can repair or improve conduction in animal heart disease models [7]

In the present review article, we aim to summarize the most up-to-date work in cell and gene therapy, and what the next steps are in making this is clinically viable option for arrhythmias.

\section{Cell mechanisms of ventricular tachycardia and ventricular fibrillation}

Ventricular tachycardia (VT) or fibrillation (VF) occur as a result of cell membrane hyperexcitability, disturbed repolarisation or defective conduction of the electrical wavefront across the myocardium [11]. Most monomorphic VTs occur in the presence of structural heart disease, caused by reentry secondary to electrically inactive scar tissue post- myocardial infarction. This forces the wavefront to propagate around a line of block, and enters the area behind the scar [6]. The non-conductive tissue creates disruptions in gap junctions and poor cell coupling, which subsequently slows conduction and/or creates a unidirectional block: these are conditions ideal for development of reentrant arrhythmias. The wave front enters an area 
behind scar, and if it reaches the back of the obstacle from multiple directions simultaneously then waves extinguish one another. However, if unidirectional block exists around one side, the wave front can reenter and re-excite tissue in front of unidirectional block, leading to stable reentry [6].

Disturbed ion channel function can also occur in structurally normal myocardium. In this instance, ventricular tachycardia is thought to arise because of triggered activity or automaticity. Both early afterdepolarizations (EADs) and delayed afterdepolarizations (DADs) can reach the threshold high enough to trigger premature ventricular contractions, these can subsequently dissipate into monomorphic or polymorphic VT [2].

\section{Cell mechanisms of bradyarrhythmias}

Reduce automaticity is the underlying mechanism in bradycardia. Conduction delay and block occurs when propagating impulse fails to conduct. One of the main causes of bradycardia is sinus node dysfunction. This causes depression of the automaticity in the sinoatrial node (SAN) and thus impairs electrical conduction from the sinus node, perinodal and atrial tissue $[12,13]$.

The most common cause of sinus node dysfunction is idiopathic degenerative fibrosis of nodal tissue that results as a normal part of the ageing process. Fibrosis leads to a loss of pacemaker cells, and a shift from central to inferior pacemaker cells within the SAN. Spontaneous diastolic depolarisation is slower in those cells leading to bradycardia [12, 13]. 


\section{Cell mechanisms of atrioventricular tachycardia.}

Atrioventricular Nodal Reentrant Tachycardia (AVNRT) is caused by a reentrant mechanism. It occurs secondary to the presence of two pathways within the AV node which have different electrophysiological properties. It occurs as the fast pathway that exists has rapid conduction and a longer refractory period in comparison to the atrioventricular node, creating a substrate for reentry. The impulse will travel through both pathways in normal conditions. In the presence of a premature stimulate, the stimulus blocks in the faster pathway due to a longer refractory period and travels through the slower accessory pathway. If slow enough, the blocked pathway can have time to recover, setting the state for reentry. In response to a premature stimulation, the stimulus can block in the fast pathway due to a longer refractory period and travel through the slow pathway. If conduction is slow enough, the blocked fast pathway can have time to recover, thus setting the stage for a reentrant circuit, translating into AV nodal tachycardia when perpetuated.

In atrioventricular nodal tachycardia (AVRT), an accessory pathway exists that has a more rapid conduction and longer refractory period in comparison to the AV node, also leading to reentry [1].

\section{Cell-based therapies}

Cell therapy offers a novel approach to arrhythmias (Table 1). Research data has shown that cell transplantation can improve or repair impulse conduction in vitro and in experimentally injured animal hearts in vivo [7]. There has been heavy focus on the use of biological pacemakers to treat bradycardias caused by SAN dysfunction or complete AV block. In this case, cells are used to enhance tissue automaticity or repair damaged conduction such as AV block to restore normal heart rhythm. For tachyarryhythmias the focus is instead to reduce myocardial excitability or repair damage to break reentrant circuits [14]. 
Proof of concept has been provided using a variety of approaches to increased inward current or decreased repolarising current as a basis for propagation of a pacemaker potential [8]. In this arena of research, both embryonic stem cells and human mesenchymal stem cells have been the main area of focus, although other cell sources (skeletal myoblasts; fibroblasts; cardiac stem cells) have been utilised. Both need electrical integration into host myocardium to allow for initiation of pacemaker activity however this does not involve further engineering because both successfully form functional gap junctions with adjacent myocytes due to abundant connexin expression [15] .

The aim of cell therapies is to restore normal function of the conduction system in the heart by transplantation of appropriate cell populations [16]. Two strategies have been explored with regards to formation of cell-based biological therapy for use in arrhythmogenesis. The first involves the injection of inexcitable cells expressing inward current that depolarises neighbouring cells to their action potential threshold. This method requires at least two cells to create a pacing unit. The second strategy, involves the injection of excitable cells with the ability to generate spontaneous action potentials. In this method, the delivered cells are the biological pacemakers. In both strategies, the assumption is that coupling will occur through gap junction formation between donor cells and between donor and target cells. Gap junctions are required for the initiation of intercellular current flow, and allowing the delivered cells to integrate electromechanically into the cardiac environment [17].

\section{Embryonic stem cells}

Use of embryonic stem cells for the treatment of bradyarrhythmias

A major barrier for the development of cell replacement strategies is the paucity of cell sources for human cardiomyocytes. A proffered solution has been human embryonic stem cells (hESCs). They are one agent being heavily utilised in the development of cell based therapies 
for cardiac arrhythmias. They are suitable in that they are pluripotent and can differentiate into any cell type, including pacemaker cells $[15,18]$. They are also clonogenic and selfpropagating [19], able to generate unlimited numbers of functional cadiomyocytes.

HESCs can differentiate in vitro and they form embryoid bodies (hEBs) composed of derivatives of all 3 embryonic germ layers. They are heterogenous, and some begin to contract spontaneously, containing cardiac myocytes that exhibit phase 4 depolarisation [20-22]. It has been demonstrated that these cardiomyoctes have the capability of forming a functional "cardiac synctium" responsive to adrenergic and cholinergic stimuli [16].

Studies demonstrate that biological pacemakers derived from hESCs are capable of pacing recipient ventricular cardiomyocytes in vitro and myocardium in vivo [23]. Cardiomyocytes derived from hESCs can electromechanically integrate with cardiomyocytes in culture. The first reported use of ESCs to create biological pacemakers was by Kehat and colleagues [24]. They showed that cardiomyocytes derived from hESCs can electromechanically integrate with cultures of neonatal ventricular myocytes. Electrograms recorded from sixty electrodes of a multi-electrode array showed initiation and conduction within the co-culture. Following addition of cardiomyocytes derived from hESCs, electrograms demonstrated tight temporal coupling between human and rat cells within one day, and lasted until the end of the experiment [25].

In further studies, it was highlighted that clusters of such cells could integrate into host myocardium in a large animal model of complete heart block, providing a proof of concept for the use of cell therapy to generate a biological pacemaker. The cells demonstrated normal cardiac excitation contraction coupling demonstrated within this tissue, electrical activation, increased in [ca2+]I and the following contraction [25].

Kehat et al. derived spontaneously-beating hEBS from cultured ESCs and implanted these into pig ventricles with complete heart block. Rhythm showed a positive chronotropic 
response upon exposure to isoproterenol administration- mimicking physiological pacemaker function. Stable pacing was observed in half; the remaining had only ectopics orginating from the site of implantation, others failed to demonstrate reliable pacing $[23,25]$. Xue et al. used a different strategy. They injected cardiomyoctes derived from hESCs into the subepicardium of the left ventricle of guinea pigs. The result was the functional integration of these cells with host tissue and to induce electrical activity to injection site to adjacent cardiac tissue [26]. It is important to note that the hosts required immunosuppression [23].

A further in vivo model was developed, whereby hEBs were injected into left ventricular anterior wall of a guinea pig [27]. A single hESC-derived hEB was transplanted on a monolayer of neonatal ventricular rat myocytes (NVRMs) serving as host. Spontaneously beating hEB and a monolayer of quiescent rat myocytes were co-cultured and demonstrated expression of gap junctions at their boundary and rhythmic contractions of the hEB. Multielectrode and optical mapping showed that spontaneous APs propagated to the NVRMS. The hESC-derived biological pacemakers spontaneously beat prior to transplantation and could be a good way to generate a spontaneous rhythm in the bradycardic setting. Immune response from the host remains the main caveat in these therapies becoming a reality, although these could be overcome by induced pluripotent stem cells [8]. Whilst recent studies have shown that hESC-CMs mature over time in culture to show a more adult phenotype, there are also concerns that ESC and induced pluripotent cell implants would lose pacing ability over time [28].

Use of embryonic stem cells in the treatment of tachyarrythmias

Whilst studies have mainly focused on the use of cell therapy for SAN damage, and AV block, their use for tachyarrythymias has also been noted, mainly post-infarct[2931]. Myocardial ischemia leads to cardiomyocyte death. Mature human cardiomyocytes 
have limited ability to regenerate, and so dead myocardium is replaced by scar tissue formed from fibroblasts; these are non-excitable with low levels of connexin expressed. As such, this scar tissue has limited capability to conduct electrical impulses leading to slowed conduction or even conduction block [32] and is essentially an arrhythmogenic focus [29]. The suggestion is that hESC-CMs can reduce the likelihood of reentrant arrhythmias under certain conditions such as post-infarct fibrosis [29].

Reentry is responsible for approximately $85 \%$ of arrhythmias that follow myocardial ischaemia or infarction [33], leading to ventricular tachycardias and fibrillation. Thompson et al. observed a decrease in incidence of re-entrant waves after engraftment of hESC-CMs in monolayers but not with the non-beating human embryoid cell engraftment [29]. This not unlike another study which showed that engraftment of eCMs in myocardial infarcted tissue reduced incidence of ventricular tachycardia in mice. It is thought that this can be attributed to a decrease in "heterogeneity of conduction" or increase in wavelength of the propagating wave. hESC CMS effectively lower resting potential of host myocytes [34].

\section{Mesenchymal Stem Cells}

Use of mesenchymal stem cells in the treatment of bradyarrythmias

Mesenchymal stem cells (MSCs) are a subset of stromal stem cells that can be isolated from many adult tissues [35]. It is suggested that they may be better than ESCs in terms of clinical application. Several trials have been performed and the possibility is that they are immunoprivileged; there have been no immune responses so far $[15,35]$. They are, in contrast to ESCs, multipotent and only able to differentiate into cells of the mesoderm lineage and as such need genetic modification in order to incorporate pacemaker properties [15].

Synchronously beating monolayers of culture neonatal rat cardiomyocytes in a MEA dish were separated by mechanically abraded channels to yield two asynchronously beating 
cardiomyocyte fields. Adding hMSCs to abraded channel resynchronised the two separated cardiomyocyte fields and conduction velocity across hMSCs increased progressively after coculture with cardiomyoctes. Cx43 expression and functional gap junctions were formed between hMSCs and cardiomyocytes with such electrical connections increasing following time after co-culture [36]. Adding hMSCs to cultures of isolated dog ventricular myocytes also formed cx43 and cx40 connections along regions cell-to cell contact, leading to cell-to cell coupling [37].

Local implantation of MSCS into the AVN area improved AV conduction in rat model with complete AVN block [38]. Animals with MSC transplantation showed significantly decreased collagen deposition in the AVN area. This is thought to potentially be due to paracrine effects of implanted MSCS and anti-fibrotic properties leading to improvement of impulse conduction. However, co-culture of hMSCs with neonatal rat ventricular myocytes decreased conduction velocity and induced reentrant arrhythmias probably due to tissue heterogeneity of inexcitable MSCs and myocytes [39]. One study showed integration could be improved: infarcted rat hearts implanted with cardiogenic cells developed from rat MSCS after treatment with a PKSC activator restored conduction velocity, by means of reducing tissue heterogenity and improving myocardial contractility [40].

\section{Use of mesenchymal stem cells in the treatment of tachyarrythmias}

The aforementioned principles have been demonstrated to show potential with regards to clinical application. Katrisis et al followed patients with history of MI with ICDs and performed intracoronary MSC and endothelial progenitor cell transplantation. Prior to stem cell transplantation, non-sustained VT inducible monomorphic VT or ventricular flutter observed in all 5 patients. At 16-36 months follow up, interrogation of ICD failed to detect any further episodes of ventricular arrhythmia, and further electrophysiological study induced 
sustained ventricular arrhythmias in only 2 patients. This however is a small, nonrandomised study, only preliminary in nature [41].

\section{Alternative cell sources}

Myoblasts

Myogenic precursor cells are also alternative sources that have been investigated. Myoblasts can be harvested from skeletal muscle biopsy. Primary myoblasts have capability of division and expansion to offer a rich cell source for transplantation. They have been transplanted autologously for myocardial repair. [42]. Ventricular tachyarrrythmias and sudden cardiac death due to failure of gap junction formation s has been observed in clinical trials with myoblast transplantation [43]. Cocultures of human skeletal myoblasts and rat cardiomyoctes reproduced reentrant arrhythmias; addition of calcium channel blocker nitrendipline blocked arrhythmias in culture [42].

\section{Interstitial cells of Cajal}

Cells termed as the interstitial cells of Cajal (ICC) are found in the human gut. They generate spontaneous pacemaker currents propagating electrical signals critical for gut peristalsis. The embryological origin of ICC are from the same embryonic germ layer mesoderm as that of heart cells [7, 44]. ICC express Cx43 and form conductive networks via these proteins [7].

Cardiac conduction repair by ICC in vitro was recently investigated. Conduction block was created by abrasion of synchronously beating monolayer of mouse atrial cells culture in MEA dish. This was co-cultured with ICC isolated from porcine ileum. Synchronous beating of cardiomyocytes was observed after addition of ICC. Evidence of in vivo success was also 
seen after injection of ICC into the right atrium. Tissue sections highlighted that ICC survived the injection two weeks later. Demonstration is that ICC could be obtained in high numbers from gut and conduct impulses across damaged cardiomyocytes. Autologous transplantation would reduce ethical issues associated with embryonic stem cells and problems with immunorejection. Further studies would need to confirm if ICCs could be used for conduction repair in vivo [7].

\section{Limiting factors}

Cell therapy offers a novel approach to treating arrhythmias. Stem cell based biological pacemakers have shown success in vivo in animal models, but are yet to meet criteria that would allow for successful translation into clinical therapy [17]. Limitations have been encountered with regards to their use for cardiac conduction damage. Whilst success has been observed in vitro and in vivo, feasibility and safety still largely unknown and remains untested. Current knowledge is based on studies of short term observation in vitro and there are still only a small number of studies that show success for conduction repair in animals [7]. Although, there is evidence of positive results from in vivo studies examining the ability of hESC- CMs to improve cardiac function, numbers of incorporated cells attributed to improvements has been low $[30,31]$. Difficulties are also faced with regards to obtaining quantitative electrophysiological assessment following hESC-CM engraftment in vivo [30, 31]. Moreover, strong concerns have also been raised regarding the pro-arrhythmic potential of cell therapies [39] [40].

Furthermore, whilst studies using ESCs have shown success there are obvious ethical concerns regarding the use of embryonic stem cells [45]. Immunogenic concerns also exist and hosts would require immunosuppression [23]. Autologous sources that bypass these problems do exist (skeletal myoblasts, pluripotent stem cells) but require genetic engineering [23]. The 
use of adult cardiac stem cells would counter these concerns however research looking at their use for biological pacing is only in its early stages [46, 47].

\section{Gene mechanisms of ventricular tachycardia and ventricular fibrillation}

As discussed, ventricular tachy-arrhythmias most often occur in the setting of structural heart disease. However, genetic disorders including LQTS, Brugada syndrome and catecholaminergic-VT, result from ion channel abnormalities and ion dysregulation in the context of a structurally normal heart [4]. LQTS is cause by a group of mutations, underpinned by a reduction in the repolarising current, thus prolonging the action potential duration [48]. The commonest forms of LQTS (types 1,2 and 3) result from loss-of-function mutations to potassium channels, and gain-of-function defects to depolarizing sodium current [48].

Brugada syndrome is endemic in east and south-east Asia. One third of cases is familial, with an autosomal dominant inheritance pattern. Loss of function of the SCN5A (sodium-carrying) channel has been identified in $20 \%$ of patients [49]. Catecholaminergic polymorphic VT is most commonly caused by a mutation to the ryanodine receptor channel, which releases calcium from the sarcoplasmic reticulum during the plateau phase of the cardiac action potential [50] .

\section{Use of Gene Therapy to treat VT/VF}

Sarcoplasmic reticulum Ca ATPase (SERCA) 2a is an ATPase pump that regulates cytosolic calcium concentration by transferring $\mathrm{Ca}$ into the lumen of the sarcoplasmic reticulum. Following ischaemia, SERCA activity decreases, increasing cytosolic $\mathrm{Ca}$ and triggering afterdepolarizations [9]. SERCA2a was overexpressed in the left ventricles of pigs who had undergone LAD occlusion and reperfusion. There was a significant reduction in arrhythmias 
of transfected animals compared with controls. There was no difference, however, in animals who had undergone artery occlusion but no reperfusion [51].

Deficient SERCA2a is also associated with cardiac failure. When transferred into the failing hearts of rats [52] and guinae pigs [53], ventricular arrhythmias were significantly suppressed. SERCA2a overexpression in the heart of healthy young guinae pigs resulted in a 4-fold reduction in alternans-mediated ventricular arrhythmias [54].

Another modulator of cytosolic calcium concentration is calsequestrin, which binds calcium within the SR. Intraperitoneal injection of WT-CASQ2 into knockout mice normalised levels of calsequestrin and its associated proteins, and significantly suppressed catecholaminergic arrhythmias [55]. A follow up study demonstrated the curative effects were seen for one year after a single vector injection [56].

Re-entry accounts for most VT/VF. In infarct epicardial border zones the cardiac sodium channel is largely inactivated contributing to slow conduction and re-entry [57]. SkM1 carries a fast inward sodium current. When injected directly into the infarct border zone, SkM1 suppressed post-infarct arrhythmias in dogs and increased Vmax of depolarised myocardium [58]. The $\mathrm{KCNH} 2$ protein forms the hERG1 channel, which mediates the repolarizing $\mathrm{K}$ current. Transfection of dominant negative mutant prolonged the refractory period and suppressed post-infarct arrhythmias in porcine models [59].

\section{Gene Mechanisms of bradyarrhythmias}

Bradycardia is generally associated with structural heart disease, but genetic cases have been identified affecting children with no underlying pathology $[5,60]$. Familial sinus bradycardia has been linked with a mutation in the HCN4 ion channel[61]. The mutant channels were 
activated at more negative voltages than the wild-type channels, decreasing the inward diastolic current and slowing the heart rate[61]. Mutations to the voltage-gated sodium channel have been linked with SCN5A-related familial sick sinus syndrome[62]

\section{Use of Gene Therapy to treat bradyarrhythmias}

Gene therapy has successfully been used to convert normally quiescent cells into pacing cells, and to increase the intrinsic rate of cells with pacing ability.

HCN (hyperpolarisation-activated cyclic nucleotide gated) channels carry the 'funny' current and are often referred to as the pacemaker channels. They are activated on hyperpolarization and allows passage of sodium and potassium. HCN4 is the main isoform found in the sinoatrial node, but low levels of $\mathrm{HCN} 1$ and $\mathrm{HCN} 2$ have been reported. As mutations to this channel have been linked with familial sinus bradycardia, several studies used HCN 'gain-of-function' to increase the heart rate of subjects.

Transfection of murine-HCN2 into the left atria of dogs results in spontaneous pacemaker activity from the left atria after vagal-stimulated sinus arrest. Importantly, the rhythm was generated from the site of injection on electrophysiological studies[63]. When HCN2 was injected into the LBB of dogs, there was a ventricular rhythm significantly faster than that seen in the controls[64].

Another group created a construct of $\operatorname{HCN} 1-\Delta \Delta \Delta$ (a channel with a shortened S3-S4 linker which favours channel opening). The recombinant adenovirus was injected into the left atria of porcine models of sick sinus syndrome. Not only did the transfected animals demonstrate a physiologically viable heart rate (average $64 \mathrm{bpm}$ ) but it responded appropriately to catecholaminergic-stimulation [65]. 
Similar viable pacemaker systems were established in rats [66] and in pigs[67] with AV block. The former study, however, showed the HCN expression only lasted for one month after injection, calling into question the long term feasibility. If the effect is only transient, it could be used for short-term 'bridging' option for patients e.g. when being treated for a device infection.

Boink et al combined HCN with adenylyl cyclase [68] and SkM1 [69] genes, to assess if the addition enhanced the pacemaker ability compared with $\mathrm{HCN}$ transfection alone. When injected into LBBB of canine models with AV block, both combinations produced a physiologically higher heart rate than $\mathrm{HCN}$ alone.

Heart rate is under control of adrenergic signalling. Edelberg et al used DNA plasmids to transfer the B2-adrenergic receptor into the right atrium of otherwise normal pigs, leading to $50 \%$ increase in heart rate, and effect that lasted 2 days[70]. Adenylyl-cyclase type 6 (ACVI) is generated in response to b-adrenergic stimulation, and then binds to $\mathrm{HCN}$ channels, where is regulates spontaneous rhythm activity. After injection into the left ventricle, all animals receiving ACVI demonstrated an escape rhythm from the site of injection of $>100 \mathrm{bpm}$ when isoprenalin was also administered. In contrast, the control animals showed an escape rhythm from the right ventricle [71].

TBX18 (transcription box factor 18) is a transcription factor that is required for SAN development in the fetal heart. Adenoviral transfer of TBX18 has successfully created ectopic pacemaker activity at sites of injection in guinae pigs [72] and pigs [73]. In the porcine model, heart rates were significantly faster in TBX18 recipients compared with controls. Furthermore, cells with SAN-morphology were detected at the sites of injection. 


\section{Gene Mechanisms of Atrial Fibrillation}

\section{Use of gene therapy to treat $A F$}

Gene therapy can be used to control the heart rate or the heart rhythm, similar to current medical therapy. During AF, normal AV conduction leads to a rapid ventricular rate and a reduction of cardiac output. Stimulation of b-adrenaline receptors results in release of Gas protein subunits, activating adenylyl cyclase. This shortens the refractory period and increases conduction through the AV node. Inhibitory G proteins have been transfected into the AV nodes of pigs, resulting in a ventricular rate reduction of $\sim 20 \%$ [74]. Rather than completely inhibiting the $\mathrm{G}$ protein, Lugenbiel attenuated the Gas with a silencing RNA (SiRNA). Again a 20\% HR was observed in transfected animals compared with controls. Alongside the reduction in heart rate, a significant increase in LVEF was observed [75].

The other medical option is rhythm control. Class III antiarrhythmic drugs work by blocking inward $\mathrm{K}$ current, and therefore prolonging the action potential and refractory period. Atrial repolarization is conducted by the delayed rectifier potassium current. Amit et al transferred a dominant-negative mutant of the underlying ether-a-go-go gene, which codes for the potassium channel. By day 10, none of the G628S recipients were in AF, but by day 21 all animals were in AF [76]. Soucek et al also achieved significant suppression of AF in pigs who were injected with adenoviral carriers [77].

$\mathrm{AF}$ is also associated with reduced expression of connexin40 and 43, which regulate conduction velocity in the heart. Adenoviral transfer of both proteins resulted in significant suppression of AF in 2 independent studies [78, 79] 


\section{Conclusions}

It has become evident that cardiac arrhythmias have a gene and cell-related substrate and those factors may well account for the presentation of different kind of arrhythmias. So far, the exact mechanisms through which genes and cells contribute to cardiac arrhythmias are not fully understood. Despite this, ongoing research using cells and genes has opened new horizons in the management of arrhythmias. The available results are promising, but still in the pre-clinical setting. Therefore, we look forward to future clinical studies, which will confirm the clinical utility of those strategies.

\section{Expert opinion}

Cardiac arrhythmias are characterised by high mortality rates especially in patients with preexisting cardiac disorders and the elderly. Conduction defects can lead to both tachycardias and bradycardias. There is also evidence that cells and genes are closely related to arrhythmias and play a significant role in arrhythmogenesis. Therefore, several studies have aimed to treat/manage arrhythmias by using cell- and gene-based or combination strategies. It appears that such therapeutic approaches can improve an impaired conduction. Recent clinical and experimental studies have assessed the efficacy of gene and cell therapies confirming potential antiarrhythmic effects. Some of the studies have shown encouraging results. However, there is still lack of large clinical trials using genes and cells to treat arrhythmias. Moreover, traditional therapeutic approaches such as drugs, devices and catheter ablation remain superior and well evaluated compared to cell and gene-based strategies. Limiting factors still remain the dosage, selection, delivery, toxicity and inflammation predisposed by those therapies. It has also been shown that some cells proliferate quickly and as a result, these cells may quickly lose the 
phenotype of the targeted gene transferred for the therapy. In addition, it is speculated that repeated gene therapy could prove to be harmful due to host immune responses, while gene vectors used currently could potentially result into different diseases. Therefore, it is evident that gene and cell-based therapeutic approaches, although promising, are still challenging and pose a risk of proarrhythmia. Future large scale studies in this field are required to evaluate the potential role of these approaches and to move from the experimental/preclinical setting to the application in the clinical practice.

Acknowledgements: none

Figure. Summary of potential effects of cell and gene-based therapies on arrhythmias.

Abbreviations: KCNE3: Potassium voltage-gated channel, Isk-related family, member 3; HERG: human Ether-à-go-go-Related Gene; SERCA: sarco/endoplasmic reticulum Ca2+ATPase

\section{Highlights box:}

1. Cardiac arrhythmias are characterised by high mortality rates especially in patients with pre-existing cardiac disorders and the elderly.

2. Genes and cells are involved in the pathogenesis of arrhythmias.

3. Gene- and cell-based strategies are promising, but still not well evaluated in the clinical setting.

4. Several limiting factors should be taken into consideration before using cell and gene therapeutic approaches in clinical practice.

5. Traditional therapeutic approaches such as drugs, devices and catheter ablation remain superior to cell- and gene-based strategies. 


\section{References:}

1. Gaztanaga, L., F.E. Marchlinski, and B.P. Betensky, Mechanisms of cardiac arrhythmias. Rev Esp Cardiol (Engl Ed), 2012. 65(2): p. 174-85.

2. Zipes, D.P., Mechanisms of clinical arrhythmias. Heart Rhythm, 2004. 1(5 Suppl): p. 4C-18C.

3. Tse, G., Mechanisms of cardiac arrhythmias. J Arrhythm, 2016. 32(2): p. 75-81.

4. Wilde, A.A. and C.R. Bezzina, Genetics of cardiac arrhythmias. Heart, 2005. 91(10): p. $1352-8$.

5. $\quad$ Spellberg, R.D., Familial sinus node disease. Chest, 1971. 60(3): p. 246-51.

6. Wagner, S., L.S. Maier, and D.M. Bers, Role of sodium and calcium dysregulation in tachyarrhythmias in sudden cardiac death. Circ Res, 2015. 116(12): p. 1956-70.

7. Xiao, Y.F., Cell and gene therapy for arrhythmias: Repair of cardiac conduction damage. J Geriatr Cardiol, 2011. 8(3): p. 147-58.

8. Zhang, H., et al., Implantation of sinoatrial node cells into canine right ventricle: biological pacing appears limited by the substrate. Cell Transplant, 2011. 20(11-12): p. 1907-14.

9. Lugenbiel, P., et al., Antiarrhythmic gene therapy - will biologics replace catheters, drugs and devices? Eur J Pharmacol, 2016. 791: p. 264-273.

10. Greener, I. and J.K. Donahue, Gene therapy strategies for cardiac electrical dysfunction. J Mol Cell Cardiol, 2011. 50(5): p. 759-65.

11. Pandit, S.V. and J. Jalife, Rotors and the dynamics of cardiac fibrillation. Circ Res, 2013. 112(5): p. 849-62.

12. Vogler, J., G. Breithardt, and L. Eckardt, Bradyarrhythmias and conduction blocks. Rev Esp Cardiol (Engl Ed), 2012. 65(7): p. 656-67.

13. Kistler, P.M., et al., Electrophysiologic and electroanatomic changes in the human atrium associated with age. J Am Coll Cardiol, 2004. 44(1): p. 109-16.

14. Itzhaki, I., et al., Calcium handling in human induced pluripotent stem cell derived cardiomyocytes. PLoS One, 2011. 6(4): p. e18037.

15. Boink, G.J., et al., Biological pacing by gene and cell therapy. Neth Heart J, 2007. 15(9): p. 318-22.

16. Gepstein, L., Y. Feld, and L. Yankelson, Somatic gene and cell therapy strategies for the treatment of cardiac arrhythmias. Am J Physiol Heart Circ Physiol, 2004. 286(3): p. H815-22.

17. Chauveau, S., P.R. Brink, and I.S. Cohen, Stem cell-based biological pacemakers from proof of principle to therapy: a review. Cytotherapy, 2014. 16(7): p. 873-80.

18. Boink, G.J. and M.R. Rosen, Regenerative therapies in electrophysiology and pacing: introducing the next steps. J Interv Card Electrophysiol, 2011. 31(1): p. 3-16.

19. Hoffman, L.M. and M.K. Carpenter, Characterization and culture of human embryonic stem cells. Nat Biotechnol, 2005. 23(6): p. 699-708.

20. Kehat, I., et al., Human embryonic stem cells can differentiate into myocytes with structural and functional properties of cardiomyocytes. J Clin Invest, 2001. 108(3): p. 407-14.

21. Mummery, C., et al., Differentiation of human embryonic stem cells to cardiomyocytes: role of coculture with visceral endoderm-like cells. Circulation, 2003. 107(21): p. 273340.

22. $\mathrm{Xu}, \mathrm{C}$., et al., Characterization and enrichment of cardiomyocytes derived from human embryonic stem cells. Circ Res, 2002. 91(6): p. 501-8. 
23. Marban, E. and H.C. Cho, Biological pacemakers as a therapy for cardiac arrhythmias. Curr Opin Cardiol, 2008. 23(1): p. 46-54.

24. Kehat, I., et al., High-resolution electrophysiological assessment of human embryonic stem cell-derived cardiomyocytes: a novel in vitro model for the study of conduction. Circ Res, 2002. 91(8): p. 659-61.

25. Kehat, I., et al., Electromechanical integration of cardiomyocytes derived from human embryonic stem cells. Nat Biotechnol, 2004. 22(10): p. 1282-9.

26. Xue, T., et al., Functional integration of electrically active cardiac derivatives from genetically engineered human embryonic stem cells with quiescent recipient ventricular cardiomyocytes: insights into the development of cell-based pacemakers. Circulation, 2005. 111(1): p. 11-20.

27. Zhang, J., et al., Functional cardiomyocytes derived from human induced pluripotent stem cells. Circ Res, 2009. 104(4): p. e30-41.

28. Sartiani, L., et al., Developmental changes in cardiomyocytes differentiated from human embryonic stem cells: a molecular and electrophysiological approach. Stem Cells, 2007. 25(5): p. 1136-44.

29. Thompson, S.A., et al., Engraftment of human embryonic stem cell derived cardiomyocytes improves conduction in an arrhythmogenic in vitro model. $\mathrm{J}$ Mol Cell Cardiol, 2012. 53(1): p. 15-23.

30. Laflamme, M.A., et al., Formation of human myocardium in the rat heart from human embryonic stem cells. Am J Pathol, 2005. 167(3): p. 663-71.

31. Leor, J., et al., Human embryonic stem cell transplantation to repair the infarcted myocardium. Heart, 2007. 93(10): p. 1278-84.

32. van den Borne, S.W., et al., Myocardial remodeling after infarction: the role of myofibroblasts. Nat Rev Cardiol, 2010. 7(1): p. 30-7.

33. Wit, A.L. and M.J. Janse, Experimental models of ventricular tachycardia and fibrillation caused by ischemia and infarction. Circulation, 1992. 85(1 Suppl): p. I3242.

34. Roell, W., et al., Engraftment of connexin 43-expressing cells prevents post-infarct arrhythmia. Nature, 2007. 450(7171): p. 819-24.

35. Rosen, M.R., et al., Genes, stem cells and biological pacemakers. Cardiovasc Res, 2004. 64(1): p. 12-23.

36. Pijnappels, D.A., et al., Progressive increase in conduction velocity across human mesenchymal stem cells is mediated by enhanced electrical coupling. Cardiovasc Res, 2006. 72(2): p. 282-91.

37. Valiunas, V., et al., Human mesenchymal stem cells make cardiac connexins and form functional gap junctions. J Physiol, 2004. 555(Pt 3): p. 617-26.

38. Yokokawa, M., et al., Transplantation of mesenchymal stem cells improves atrioventricular conduction in a rat model of complete atrioventricular block. Cell Transplant, 2008. 17(10-11): p. 1145-55.

39. Chang, M.G., et al., Proarrhythmic potential of mesenchymal stem cell transplantation revealed in an in vitro coculture model. Circulation, 2006. 113(15): p. 1832-41.

40. Song, H., et al., Cardiomyocytes from phorbol myristate acetate-activated mesenchymal stem cells restore electromechanical function in infarcted rat hearts. Proc Natl Acad Sci U S A, 2011. 108(1): p. 296-301.

41. Katritsis, D.G., et al., Transcoronary transplantation of autologous mesenchymal stem cells and endothelial progenitors into infarcted human myocardium. Catheter Cardiovasc Interv, 2005. 65(3): p. 321-9. 
42. Menasche, P., et al., The Myoblast Autologous Grafting in Ischemic Cardiomyopathy (MAGIC) trial: first randomized placebo-controlled study of myoblast transplantation. Circulation, 2008. 117(9): p. 1189-200.

43. Macia, E. and P.A. Boyden, Stem cell therapy is proarrhythmic. Circulation, 2009. 119(13): p. 1814-23.

44. Takaki, M., Gut pacemaker cells: the interstitial cells of Cajal (ICC). J Smooth Muscle Res, 2003. 39(5): p. 137-61.

45. Robertson, J.A., Embryo stem cell research: ten years of controversy. J Law Med Ethics, 2010. 38(2): p. 191-203.

46. Bolli, R., et al., Cardiac stem cells in patients with ischaemic cardiomyopathy (SCIPIO): initial results of a randomised phase 1 trial. Lancet, 2011. 378(9806): p. 1847-57.

47. Makkar, R.R., et al., Intracoronary cardiosphere-derived cells for heart regeneration after myocardial infarction (CADUCEUS): a prospective, randomised phase 1 trial. Lancet, 2012. 379(9819): p. 895-904.

48. Clancy, C.E. and R.S. Kass, Inherited and acquired vulnerability to ventricular arrhythmias: cardiac Na+ and K+ channels. Physiol Rev, 2005. 85(1): p. 33-47.

49. Wilde, A.A., et al., Proposed diagnostic criteria for the Brugada syndrome. Eur Heart J, 2002. 23(21): p. 1648-54.

50. Kontula, K., et al., Catecholaminergic polymorphic ventricular tachycardia: recent mechanistic insights. Cardiovasc Res, 2005. 67(3): p. 379-87.

51. Prunier, F., et al., Prevention of ventricular arrhythmias with sarcoplasmic reticulum Ca2+ ATPase pump overexpression in a porcine model of ischemia reperfusion. Circulation, 2008. 118(6): p. 614-24.

52. Lyon, A.R., et al., SERCA2a gene transfer decreases sarcoplasmic reticulum calcium leak and reduces ventricular arrhythmias in a model of chronic heart failure. Circ Arrhythm Electrophysiol, 2011. 4(3): p. 362-72.

53. Cutler, M.J., et al., Targeted sarcoplasmic reticulum Ca2+ ATPase 2 a gene delivery to restore electrical stability in the failing heart. Circulation, 2012. 126(17): p. 2095-104.

54. Cutler, M.J., et al., Targeted SERCA2 a gene expression identifies molecular mechanism and therapeutic target for arrhythmogenic cardiac alternans. Circ Arrhythm Electrophysiol, 2009. 2(6): p. 686-94.

55. Denegri, M., et al., Viral gene transfer rescues arrhythmogenic phenotype and ultrastructural abnormalities in adult calsequestrin-null mice with inherited arrhythmias. Circ Res, 2012. 110(5): p. 663-8.

56. Denegri, M., et al., Single delivery of an adeno-associated viral construct to transfer the CASQ2 gene to knock-in mice affected by catecholaminergic polymorphic ventricular tachycardia is able to cure the disease from birth to advanced age. Circulation, 2014. 129(25): p. 2673-81.

57. Lue, W.M. and P.A. Boyden, Abnormal electrical properties of myocytes from chronically infarcted canine heart. Alterations in Vmax and the transient outward current. Circulation, 1992. 85(3): p. 1175-88.

58. Lau, D.H., et al., Epicardial border zone overexpression of skeletal muscle sodium channel SkM1 normalizes activation, preserves conduction, and suppresses ventricular arrhythmia: an in silico, in vivo, in vitro study. Circulation, 2009. 119(1): p. 19-27.

59. Sasano, T., et al., Molecular ablation of ventricular tachycardia after myocardial infarction. Nat Med, 2006. 12(11): p. 1256-8.

60. Bharati, S., et al., Familial congenital sinus rhythm anomalies: clinical and pathological correlations. Pacing Clin Electrophysiol, 1992. 15(11 Pt 1): p. 1720-9. 
61. Milanesi, R., et al., Familial sinus bradycardia associated with a mutation in the cardiac pacemaker channel. N Engl J Med, 2006. 354(2): p. 151-7.

62. Gui, J., et al., Multiple loss-of-function mechanisms contribute to SCN5A-related familial sick sinus syndrome. PLoS One, 2010. 5(6): p. e10985.

63. Qu, J., et al., Expression and function of a biological pacemaker in canine heart. Circulation, 2003. 107(8): p. 1106-9.

64. Plotnikov, A.N., et al., Biological pacemaker implanted in canine left bundle branch provides ventricular escape rhythms that have physiologically acceptable rates. Circulation, 2004. 109(4): p. 506-12.

65. Tse, H.F., et al., Bioartificial sinus node constructed via in vivo gene transfer of an engineered pacemaker HCN Channel reduces the dependence on electronic pacemaker in a sick-sinus syndrome model. Circulation, 2006. 114(10): p. 1000-11.

66. Zhong, Y.M., et al., [Transfecting rat heart with human pacemaker gene in vivo to create a biological pacemaker]. Zhonghua Yi Xue Za Zhi, 2006. 86(40): p. 2831-5.

67. Cai, J., et al., Adenoviral gene transfer of HCN4 creates a genetic pacemaker in pigs with complete atrioventricular block. Life Sci, 2007. 80(19): p. 1746-53.

68. Boink, G.J., et al., Ca(2+)-stimulated adenylyl cyclase AC1 generates efficient biological pacing as single gene therapy and in combination with HCN2. Circulation, 2012. 126(5): p. 528-36.

69. Boink, G.J., et al., HCN2/SkM1 gene transfer into canine left bundle branch induces stable, autonomically responsive biological pacing at physiological heart rates. $\mathrm{J}$ Am Coll Cardiol, 2013. 61(11): p. 1192-201.

70. Edelberg, J.M., et al., Molecular enhancement of porcine cardiac chronotropy. Heart, 2001. 86(5): p. 559-62.

71. Ruhparwar, A., et al., Adenylate-cyclase VI transforms ventricular cardiomyocytes into biological pacemaker cells. Tissue Eng Part A, 2010. 16(6): p. 1867-72.

72. Kapoor, N., et al., Direct conversion of quiescent cardiomyocytes to pacemaker cells by expression of Tbx18. Nat Biotechnol, 2013. 31(1): p. 54-62.

73. Hu, Y.F., et al., Biological pacemaker created by minimally invasive somatic reprogramming in pigs with complete heart block. Sci Transl Med, 2014. 6(245): p. 245 ra94.

74. Donahue, J.K., et al., Focal modification of electrical conduction in the heart by viral gene transfer. Nat Med, 2000. 6(12): p. 1395-8.

75. Lugenbiel, P., et al., Genetic suppression of Galphas protein provides rate control in atrial fibrillation. Basic Res Cardiol, 2012. 107(3): p. 265.

76. Amit, G., et al., Selective molecular potassium channel blockade prevents atrial fibrillation. Circulation, 2010. 121(21): p. 2263-70.

77. Soucek, R., et al., Genetic suppression of atrial fibrillation using a dominant-negative ether-a-go-go-related gene mutant. Heart Rhythm, 2012. 9(2): p. 265-72.

78. Igarashi, T., et al., Connexin gene transfer preserves conduction velocity and prevents atrial fibrillation. Circulation, 2012. 125(2): p. 216-25.

79. Bikou, O., et al., Connexin 43 gene therapy prevents persistent atrial fibrillation in a porcine model. Cardiovasc Res, 2011. 92(2): p. 218-25.

80. Bauer, A., et al., Inhibitory $G$ protein overexpression provides physiologically relevant heart rate control in persistent atrial fibrillation. Circulation, 2004. 110(19): p. 311520.

81. Murata, M., et al., Creation of a genetic calcium channel blocker by targeted gem gene transfer in the heart. Circ Res, 2004. 95(4): p. 398-405. 
82. Trappe, K., et al., Suppression of persistent atrial fibrillation by genetic knockdown of caspase 3: a pre-clinical pilot study. Eur Heart J, 2013. 34(2): p. 147-57. 